\title{
Wounded healers
}

Doctors do on average live a bit longer than the general population, primarily because they are richer and don't smoke. But they experience twice the rates of burnout, stress-induced emotional and mental illness, and addiction to drugs and alcohol. ${ }^{1}$ The noble attributes of resilience and self-reliance serve doctors well until, it seems, they fall ill. Then, hindered by a need to portray a healthy image to all around, the barriers go up and they try toughing it out.,3 Doctors, especially GPs, tend to avoid or delay asking for medical help. ${ }^{4}$ They take less sick leave, but when they do go sick it is for longer. ${ }^{5}$ They may start to self-diagnose and then to self-treat. For hospital doctors there are the added pitfalls of corridor consultations and ready access to specialist opinions and investigations.

Many of the barriers to accessing health care which doctors experience are no different from those met by any patient: embarrassment, fear, hassles of time and cost, loss of control, and oscillating between the panic of imminent disaster and the guilt of a trivial worry. There are some special barriers that doctors face: their prior knowledge of the health system and personnel; possible concerns about the quality of care; difficulty finding the right GP; and confidentiality, especially in hospitals. ${ }^{3,5-8}$

These observations are not UK or NHS specific. ${ }^{5}$ They have been similarly reported in the US, Canada, Switzerland, Australia, New Zealand, the Netherlands, Norway, Ireland, Israel, and Finland. But doctors in the UK have one special advantage: over $90 \%$ are personally registered with a GP, compared, for example, with $21 \%$ in Switzerland.

Spiro and Mandell wrote tenderly about the stories they gathered from the sick doctors - 'wounded healers' - they talked with. ${ }^{3}$ Doctors became exquisitely sensitive to the reality of their own symptoms. For them, emotion flooded back into medicine as they learned the comfort that comes from attention and devotion. 'Immigrants' to the nation of patients, they were grateful for minor details, and the kindness of strangers turned friends and caretakers. Doctors became acutely aware of the side effects of drugs, noisy wards at night, gowns that don't do up at the back. Sweeney et al and Stowell, working GPs, have separately written about their personal experiences of being diagnosed and treated for cancer in their fifties. A hackneyed turn of phrase unexpectedly hit a raw nerve; ${ }^{9}$ and an interest to see the wound made all the difference. ${ }^{10}$

Fox et $a /^{11}$ posed a similar question 'What happens when doctors are patients?', reported in this month's BJGP. They interviewed 17 UK GPs who had experienced significant illness, and confirmed that the ethos of invulnerability to illness persists among GPs. Some of the GPs also felt their role as a doctors' doctor could be awkward and ambiguous.

The General Medical Council (GMC) has a section on health in Good Medical Practice. ${ }^{12}$ The GMC states that as a doctor you should register with an independent GP outside your family; get immunised against common serious infections; and if you are ill see a doctor, follow their advice, and do not treat yourself or rely on your own assessment. The British Medical Association's (BMA's) guidance ${ }^{13}$ on the ethical responsibilities in treating doctors who are patients covers: treating family or friends, addressing doctors' health problems, self-diagnosis and treatment, responsibilities of colleagues, confidentiality, charging colleagues, mental illness, dealing with addiction, and guidance for doctors suffering from infectious diseases.

There are services available to sick doctors beyond those offered by the NHS. The BMA Counselling Service is staffed 24 hours by counsellors; and there is an enhanced service, Doctors for Doctors, where a volunteer doctor is available to give advice. Both are for doctors in distress or difficulty. The Sick Doctors Trust is a national charity for advice and support for doctors suffering from addiction to drugs or alcohol. It too runs a support line round the clock. For GPs, local medical committees and faculties of the Royal College of General Practitioners run peer support schemes. Primary care trusts operate procedures for doctors in difficulty, which combine managing poor performance with support for sick doctors.

But none of this really gets to the root of the beliefs, attitudes, and behaviour patterns - the culture - of doctors 'who made an unconscious pact with the Creator: we will take care of the sick and You will guarantee us good health'. ${ }^{3}$ The evidence suggests that this culture puts doctors exposed to high levels of distress and suffering of patients, and abuse from patients, at extra risk of mental illness and addiction. ${ }^{1}$

The first task for any UK doctor is to put the current GMC and BMA advice into practice. Have you ever discussed this guidance with colleagues? Would a review of the arrangements you make for sickness cover and locum insurance be helpful? When would you ask a medical colleague in your practice to undergo an occupational health assessment before returning to work after sickness?

Then it gets more tricky: knowing what to do is not the same as being attuned to doing it when the moment comes. Apart from the usual calls for more research, ideas from the literature centre on occupational health services; educational efforts to change attitudes; and novel changes of style for established doctors.

It is UK Department of Health policy that primary care organisations should provide occupational health services to all GPs and their staff. ${ }^{14}$ Although services remain patchy, insecure, and unsatisfactory in their provision and funding, ${ }^{15}$ a skilled occupational physician will provide an impartial opinion to both parties about the realities and options of returning to work, when vested interests can cloud a decision. They may arrange out-of-area referrals to overcome local problems of recognition and confidentiality.

Being seriously ill is certainly one very nasty way to learn what patients 
experience and need from their doctors. Doctors who have been unfortunate enough to learn in this way can use their experience to share their learning. ${ }^{3,7,9,10}$ The medical humanities, through literature and the arts, can provide a short cut for students to acquire valuable exposure to suffering, relationships, and illness. ${ }^{16,17}$ Group work and experiential learning can fill the gaps left by intellectual learning.

Spiro and Mandell suggested four style changes, refreshingly non-PC, for treating doctor-patients which are based on insights gained in their research. ${ }^{3}$ Forget autonomy: most sick doctors were thankful and relieved when another doctor took over their care. Second, spare them the detail: doctor-patients took trust and loyalty as read. Try being a bit paternalistic ('parentalisitic' is the word the Americans use). Many sick doctors expressed the peace of mind that came when they no longer had the task of making one decision after another, not least because they knew they weren't thinking straight. Last, sick doctors yearned for compassion, kindness, faith, and hope. Consolation and optimism were as important as the hard factual truths, which weren't always welcome. And if that's what doctors want, it could just be that all our patients would take relief, comfort, and hope from such a style of doctoring.

\section{Nick Bradley,}

GP Principal, Ide Lane Surgery, Alphington, Exeter.

\section{Provenance}

Commissioned; not peer reviewed.

\section{REFERENCES}

1. British Medical Association. Doctors' health matters. the work of the doctors for doctors unit in supporting doctors and promoting wellbeing within the profession. A report by the Health Policy \& Economic Research Unit. London: BMA, 2007.

2. Thompson WT, Cupples ME, Sibbett $\mathrm{CH}$, et al. Challenge of culture, conscience, and contract to general practitioners' care of their own health: qualitative study. BMJ 2001; 323(7315): 728-731.

3. Spiro HM, Mandell HN. When doctors get sick. Ann Int Med 1998; 128(2): 152-154.

4. Davidson SK, Schattner PL. Doctors' health-seeking behaviour: a questionnaire survey. Med J Aust 2003; 179(6): 302-305.

5. Kay M, Mitchell G, Clavarino A, Doust J. Doctors as patients: a systematic review of doctors' health access and the barriers they experience. Br J Gen Pract 2008; 58(552): 501-508.

6. McKevitt C, Morgan M. Illness doesn't belong to us. J $R$ Soc Med 1997; 90(9): 491-495.

7. Klitzman R. Improving education on doctor-patient relationships and communication: lessons from doctors who become patients. Acad Med 2006; 81(5): 447-453.

8. Kay MP, Mitchell GK, Del Mar C. Doctors do not adequately look after their own physical health. Med J Aust 2004; 181(7): 368-370.

9. Sweeney K, Toy L, Cornwell J. A patient's journey. Mesothelioma. BMJ 2009; 339: b2862.

10. Stowell G. 'Would you like to see the wound?' BMJ 2008; 337: a 1064

11. Fox F, Harris M, Taylor G, et al. What happens when doctors are patients? Qualitative study of GPs. $\mathrm{Br} J$ Gen Pract 2009; 59(568): 811-818.

12. General Medical Council. Good medical practice. London: GMC, 2006.

13. British Medical Association. Ethical responsibilities in treating doctors who are patients. London: BMA, 2004

14. Department of Health. The provision of occupational health and safety services for general medical practitioners and their staff. London: Department of Health, 2001

15. Harrison J. Illness in doctors and dentists and their fitness for work - are the cobbler's children getting their shoes at last? Occup Med 2006; 56(2): 75-76.

16. Skelton JR, Thomas CP, Macleod JA. Teaching literature and medicine to medical students, part I: the beginning. Lancet 2000; 356(9245): 1920-1922.

17. Skelton JR, Macleod JA, Thomas CP. Teaching literature and medicine to medical students, part II: why literature and medicine. Lancet 2000; 356(9246): 2001-2003.

DOI: $10.3399 /$ bjgp09X472836

\section{ADDRESS FOR CORRESPONDENCE}

Nick Bradley

Ide Lane Surgery, Alphington,

Exeter EX2 8UP.

E-mail: nickbradley@eclipse.co.uk 